\title{
Hemoperitoneum from suspected uterine perforation: Nonsurgical management of a case of choriocarcinoma. A case report
}

\author{
Pramila Pradhan \\ Prof and Head Department of Ob/Gyn \\ Nepal Medical College Teaching hospital
}

\begin{abstract}
A case of choriocarcinoma presented as an acute abdomen because of intraperitoneal haemorrhage from uterine perforation, 4 weeks after second and 11 weeks following first suction evacuation with high serum hCG level and metastases to vagina and lungs.

Once the uterine perforation occurs following gestational trophoblastic neoplasia, immediate laparotomy has been performed usually followed either by local resection or hysterectomy depending on situation.

This case is an exception, which was conservatively managed by chemotherapy, EMA-CO regime successfully with no remission on follow up.
\end{abstract}

Keywords: choriocarcinoma, intraperitoneal haemorrhage, uterine perforation.

\section{Introduction}

Gestational choriocarcinoma is an oppressive neoplasia arising in the body of the uterus and a feared complication following complete hydatidiform. Histologically, it is characterized by sheets of anaplastic syncytiotrophoblasts and cytotrophoblasts without chorionic villi. Unfortunately, overlooking such pathognomic feature in a case, led to an emergency admission because hemoperitoneum and acute abdomen with full blown metastatic disease into lungs/ vagina is reported herewith. Similar features of hemoperitoneum was subsequent to uterine perforation in choriocarcinoma. ${ }^{1}$

\section{Case Report}

A 22 years old was admitted on $26^{\text {th }}$ April 2006, with history of acute abdomen of half an hour duration, scanty vaginal bleeding on and off for one month and haemoptysis of 12 day's duration. The patient gave history of molar evacuation of 14 weeks gestation 11 weeks ago in a tertiary care hospital. A repeat or $2^{\text {nd }}$ evacuation had been done 4 weeks ago for severe vaginal bleeding in the same hospital. Both the times, histological report revealed trophoblastic cells exhibiting moderate cellular atypia, no chorionic villi, or hydropic villi seen. On these two occasions, she had been asked for follow up visit with urinary hCG level 2 weeks later which was not valued by her.

On admission, she was in agony with pain; her general condition was low with gross pallor, Pulse $92 / \mathrm{min}$ and regular, Blood pressure $90 / 60 \mathrm{~mm}$ of $\mathrm{Hg}$, respiration rate $26 / \mathrm{min}$. The respiratory and cardiovascular system revealed no abnormality on clinical examination. Abdominal examination revealed generalized tenderness with guarding and rebound tenderness but no definite mass or lump was felt. Per vaginal examination revealed bluish tender suburethral nodule size $3 \mathrm{~cm} \mathrm{X} 3 \mathrm{~cm}$. Speculum examination revealed healthy cervix through which brownish blood was coming. Bimanual examination revealed a uterus enlarged to 10 weeks size, soft but tender. The fornices were clear and non tender.

Haemoglobin level was 9.7 gm\%, urinary pregnancy test was positive. Xray chest PA view detected nodular

Correspondence

Pramila Pradhan. MRCOG

Prof and Head Department of Ob/Gyn

Nepal Medical College Teaching hospital Attarkhel, Kathmandu, Nepal

Email:drpramilapradhan@yahoo.com 
opacities in lower zones of both the lungs (? secondaries), sputum for AFB and malignancy were negative. Serum b hcG level was reported as 96905 $\mathrm{mIU} / \mathrm{ml}$. Ultrasonogram of upper abdomen was normal and the uterus was bulky (10.7 X 7.3 X $5.8 \mathrm{~cm})$. There was mixed echoic area with small cystic lesions filling the endometrial cavity with irregular outline invading the whole thickness of myometrium with high vascularity and neovascularisation. There was collection of fluid in the pelvic cavity which on aspiration was frank blood.

With the above findings, the diagnosis of metastatic choriocarcinoma with uterine perforation was made and staged as III FIGO placing in WHO scoring system at high risk category. She was resuscitated with oxygen inhalation, intravenous antibiotics, and two units of $\mathrm{O}$ positive blood transfused and prepared for laparotomy and shifted in the operation table approximately that took 8 hours since admission. The operation was postponed as patient was stable and to avoid hysterectomy at such a young age. She was transferred to intensive care unit for close monitoring and immediate administration of chemotherapy, EMA-CO regime with preparedness for laparotomy if there was a need. To our surprise, the patient's response to the treatment was remarkable. EMA-CO regime was continued as scheduled. Metastases in lungs and vagina disappeared after $3^{\text {rd }}$ course of chemotherapy and serum $b$ hCG level came to normal after $5^{\text {th }}$ course of chemotherapy. Two more courses of chemotherapy was given after serum b hCG became normal. She is on regular follow-up with serum b hCG every three monthly and it is clinically undetected so far.

\section{Discussion}

On the basis of medline search, to our knowledge this is the first reported case of choriocarcinoma with suspected spontaneous uterine perforation and intraperitoneal haemorrhage, treated by chemotherapy immediately avoiding laparotomy. However perforation have resulted iatrogenically at the time of second curettage in $4 / 85$ cases of persistent trophoblastic disease or after a molar evacuation. ${ }^{2,3}$

In order to stop malignant transformation or progression of molar to choriocarcinoma, chemotherapy must be advocated whenever hCG regression pattern is not observed. Rise, plateau or non decline of serial hCG in desired time is prognostic indicator of persistent disease. This requires great compliance between patients and health care providers. This woman did not come for follow up twice with urinary hCG report.
She also underwent curettage twice. A Dutch ${ }^{2}$ study showed debulking effect of the second curettage in few patient, that is $8 / 85$ where the curettage obviated the need of chemotherapy against 229 women in similar low- risk persistent trophoblastic disease (PGD, who did not undergo curettage but all of them needed chemotherapy $((\mathrm{P}<0.001)$. Unfortunately four of the eight women sustained uterine perforation at curettage which was also conservatively managed. Therefore the second curettage has some proven benefit for having lesser chance of needing chemotherapy but equal possibility to be complicated by uterine perforation. Actually many do not favor second curettage at all or is rather contradicted, since a high proportion of the patients with PGD will ultimately need chemotherapy because of the deeper myometrial invasion or molar proliferation and pelvic metastases.

In this case, uterine perforation was probably caused by rapid growth and myometrial invasion but not following curettage but the likelihood of this condition must always be kept in mind. Spontaneous uterine perforation have occurred after invasive mole, placental site trophoblastic tumor and choriocarcinoma. ${ }^{4-7}$

Choriocarcinoma metastasize readily. It is not uncommon to find secondary nodule in the cervix, vagina or vulva. ${ }^{7}$ They are easily detected and form purple haemorrhagic projections. Their appearance is characteristic and pathognomic of choriocarcinoma. Multiple metastases may form in the lungs and cause haemoptysis. These both characteristics were present in our patient. Besides these, gastrointestinal tract, liver or brains are other distant organs involved. ${ }^{7}$ Source of hemoperitoneum being bleeding from hepatic metastases in a puerperal case. ${ }^{8}$

Hysterectomy were done in large number of cases, 16 out of 27 gestational trophoblastic neoplasia because of uterine perforation. ${ }^{1,7}$ Lately localized resection of uterus has been considered in women who are desirous of future fertility followed by successful term pregnancies after such surgery. ${ }^{9,} 10$

This woman in FIGO stage III, with high score on WHO Prognostic Scoring system was effectively treated by EMA-CO chemotherapeutic regimen with remarkable response.

In this case, we presume that the patient had small uterine perforation with intraperitoneal haemorrhage which might have sealed on its own. Resuscitative measures and proper vigilance saved her from undergoing hysterectomy, a drastic procedure in young girls. Such conservative approach has also been adopted in four cases of PGD with uterine perforation. 


\section{Conclusion}

Mole progressing to chroriocarcinoma within short time is seen to have favorable prognosis if the right decision is timely taken adhering to a saying "a stitch in time saves nine." Besides proper evaluation of histpathological report must be realized equally important as is the follow up with serum bhCG to avoid acute emergency like shock and intraperitoneal hemorrhage.

\section{References}

1. Chan ST, Wee D, Lim- Tansk. Choriocarcinoma following term pregnancy: a case report. Ann Med Singapore 1990; 19 (3): 421-22.

2. Trommel NE, Massuger LF, Verheijen RH, Sweep FC, Thomas CM. The curative effect of a second curettage in persistent trophoblastic disease: a retrospective cohort survey. Gynecol Oncol. 2005 Oct; 99(1):6-13.

3. Manandhar B, Rana A, Pradhan N, Sharma R. Uterine perforation as a sequele to repeated curettage for molar pregnancy. JNMA 2002, 4:40103 .

4. Kumar S, Vimala N, Mittal S. Invasive mole presenting as acute haemoperitoneum. JK Science. 2004; 6:3:1-3.
5. Chandran R, Tham KY, Rose I. Perforating invasive mole masquerading as an ovarian tumour-case report. Med J Malaysia. 1991 Sep; 46(3):255-8.

6. Mehta S, Gehlot M. Choriocarcinoma leading to uterine perforation: a case report. The Journal of Obstetrics and Gynecology of India. 2002 MarApr; 52(2): 142

7. Xiang Y, Yang X, Zhang L, Song H. Evaluation of emergency surgery in gestational trophoblastic tumours. 1997 Oct; 9(5):369-72.

8. Erb RE, Gibler WB. Massive hemoperitoneum following rupture of hepatic metastases from unsuspected choriocarcinoma. Am J Emerg Med. 1989 Mar;7(2):196-8.

9. Behtash N, Ansari S, Saroi F. Successful pregnancy after local resection of perforated uterus in choriocarcinoma and a literature review. Int. J gynaecol cancer 2006; 16 (supp1): $445-48$.

10. Lin HW, Shieh CS, Chen LM, Yang YT, Han CP. Spontaneous uterine perforation mimicking ectopic pregnancy as the initial presentation of placental site trophoblastic tumor. Zhonghua Yi Xue Za Zhi (Taipei). 2001 Sep; 64(9):545-50. 